\title{
A Binocular Display Augmented Reality Smart Helmet
}

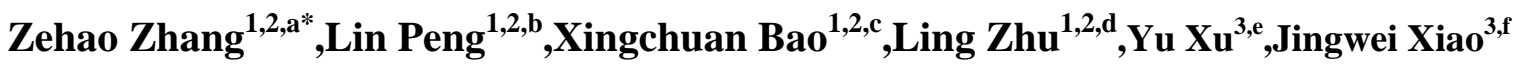 \\ ${ }^{1}$ Global Energy Interconnection Research Institute co.,Ltd Research Institute of Information \\ Technology\&Communication, Nanjing, 210003, China;
}

${ }^{2}$ State Grid Laboratory of Electric Power Communication Network Technology, Beijing, 102209, China;

${ }^{3}$ State Grid Chongqing electric power company, ChongQing, 400000, China.

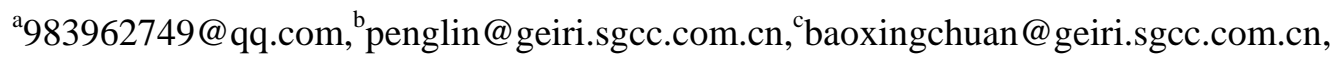

dzhuliang@geiri.sgcc.com.cn, ${ }^{\mathrm{e}}$ xuyu@geiri.sgcc.com.cn, ${ }^{\mathrm{f} x i a o j i n g w e i @ g e i r i . s g c c . c o m . c n ~}$

\begin{abstract}
The intelligent dressing equipment, the important development direction of future mobile computing form smart helmet is one of the important equipment of intelligent wear equipment form, it is a multidisciplinary integrated embodiment of cutting-edge technology.This article mainly introduces the design process of smart helmet, the main function and the development of key steps, and shows the finished product of the smart helmet.
\end{abstract}

Keywords: Binocular display, Augmented reality, Smart helmet, Development process.

\section{Introduction}

Current IT domain wearable technology and product development rapidly, a large number of virtual reality, smart, intelligent bracelet watches and other products for the consumer market emerge in endlessly, mainly used for the industrial market has enhanced display intelligent helmets, intelligent glasses, etc.The application of industrial grade wearable devices in the power industry can effectively improve the informatization and intelligence level of the on-site operations such as the production and manufacturing of power equipment, infrastructure and installation, operation and maintenance, etc.The industrial-grade smart helmet mainly foreign Hololens, Daqri, Atheer, and other products, but the direct application of external products, there is expensive for popularization and application, the power industry proprietary business pertinence is not strong, some problems such as safety, so the need for independent intellectual property rights of the grid work smart helmet equipment development.

\section{Intelligent helmet development process.}

According to the development process of hardware products, the development process of hardware products is mainly divided into the following steps:

(1) Market research

Market research includes three aspects: 1) understand the market demand.Understand how much of the same product is in the current market, and the price, specification and other information of the product.And understand the current market demand for the product, and the development of the situation.The market outlook is good.2) understand customer requirements.Communicate with the customer to understand what the customer's requirements are and what the requirements are for the performance of the product.3) analyze customer requirements and transform into customer 
requirements.Summarize customer's requirement analysis and translate it into customer demand.

(2) Project approval.

First, the requirements of the project need to be clearly defined;The time required to complete the project;Departments that need cooperation;Estimated cost;The functions and specifications of each part of the project, and then clarify their respective tasks with relevant departments.After the approval of the general manager, the general manager approved the project and began to develop the project.If there is an accident, it is necessary to modify the contents of it, and it needs to be discussed with the relevant departments. After the general engineer agrees, the general manager shall revise it after approval.

(3) Overall hardware design.

The overall hardware design includes: dividing the hardware part of the project into modules, specifying the function of each module, completing the time and responsible person;Communication and connection between blocks;Power requirements;Communication protocol;The main part of the project and the difficult part of the attack time and so on.This step is to make overall planning for the whole project, and need to have overall assurance of the project.Reasonable and effective arrangement of each task.

(4) The selection of core devices and detailed design of sub-modules.

After the overall design is completed, the core components need to be selected and the design scheme of module is started.The design plan needs to be clear: this module functions, completes the time, the responsible person;Communication protocol;Number of interfaces;Design principles and other details.If special circumstances need to be modified, it will be modified by relevant personnel.The experiment of the core device needs to leave the experiment report, record the time, place, cooperate the personnel, the experiment process, the experiment result, the experiment conclusion analysis and so on.

(5) Circuit, procedure and appearance design.

After the detailed design of the selection and sub-module of the core device is completed, the circuit, program and appearance design are carried out.

The circuit design needs to complete schematic diagram design, PCB diagram production, research and development materials, circuit debugging report, debugging manual, hardware test document, etc.After the circuit diagram design is completed, after the debugging is normal, the research and development materials and hardware test documents are generated.In the process of circuit design, it may be necessary to have multiple boards.After the circuit board production is finished, make the experiment board.The debugging of the test board may need to be coordinated with the program.The final debugging is completely in accordance with the requirements of the design plan, and the function is complete, that is, the circuit board design is completed.In each version of circuit board debugging, the debugging report needs to be completed.Summarize the problems in circuit board debugging.Convenient for the next version of the circuit board modification.

The program design needs to complete the program burning files, program modification documents.After the program is completed, the software test document is generated.The software 
test documentation should indicate program test conditions, test procedures, tools needed, test priorities, requirements for testing, etc.After approval, modify version number and merge into file.

The appearance design needs to complete the CAD drawing document and assemble the mechanical material list.After the CAD drawing is completed, the assembly machine material is generated.The list of assembly machines shall be marked: project name, mechanical drawing name, version number;Assembly tools; The number of assembly components, manufacturer, manufacturer, description and other information. When the material is finished, enter the file.The entry name should contain the version number.If the material list is modified, after approval, modify version number and merge into file.

(6) System coordination.

After each module is debugged, the system can be combined.The system coordination may require the cooperation of various departments, write the plan ahead of time, list the time of system intermodulation, test the project, cooperate with the personnel and so on. The system coordination report needs to be completed.In the report, it is necessary to record the steps of the intermodulation, the problems and solutions in the process, and the time to solve the problem.

\section{Main functions of smart helmet.}

Enhance the reality display function: realize the reality display of binocular enhancement, the transmittance is over $60 \%$, the horizon is over 30 , and the resolution is above $720 \mathrm{p}$, which can display the content in outdoor environment.It is mainly realized in the front of the helmet, close to the eye, up and down, using the lumus ultra-thin display optical module, and the semi-transparent enhancement of the reality.

Data storage, processing and transmission functions: the overall data processing performance reaches the level of high-end mobile devices in the industry.Meet the demand of data processing such as display and sensor in power operation environment.To store all kinds of data of grid operation requirements, meet the local data needs of work, and support the external expansion of data storage.It can meet the demand of data transmission and data transmission of audio and video data, sensor data and so on.

Human-computer interaction: to realize human-computer interaction, including speech recognition interaction and gesture interaction.Implementation: the upper part of the helmet, using the depth camera, is realized through the human-computer interaction middleware.

Multimedia input and output function: shooting (recording) clear images to meet the image input requirements.Audio input and output can be performed in the industrial environment of grid operation.Implementation: helmet front camera, side microphone and headset implementation.

Software system function: based on android's open system, it provides hardware module driver and can deploy power grid operation application.Implementation: customize android.

Safety reinforcement function: meet the requirement of secure access to the mobile device of the power grid.Implementation: the encryption algorithm is implemented in the Android system and the Linux underlying software, and the hardware is implemented in the encrypted TF card.

Man-machine engineering and reliability: meet the requirements of ergonomics and industrial reliability in the industrial environment of power grid.Implementation method: design helmet by 
ergonomics method, and control the helmet in accordance with the reliability principle.

\section{Develop key steps and display of finished products.}

On the basis of detailed design scheme of electronic product development processes, design of main board schematic and PCB figure, after the design review, after review the sample plate, processing, finished components at the same time, the procurement of pattern welding, detect and correct after electricity debugging, find and analyze problems after modified design, design review, again again sample plate, machining, welding, so repeatedly, until, after debugging found no problem on sample assembly, forming prototype;After the test of the non-developer of the prototype, if the hardware problem is found, the schematic diagram and PCB design need to be modified and re-evaluated.If the software problem is found, the bug can be modified so that it can be tested in a small batch until the problem is not detected by the non-developer test.So repeatedly, gradually improve the trial production, reduce the development risk.The trial process of the prototype intelligent helmet is shown in figure 1.

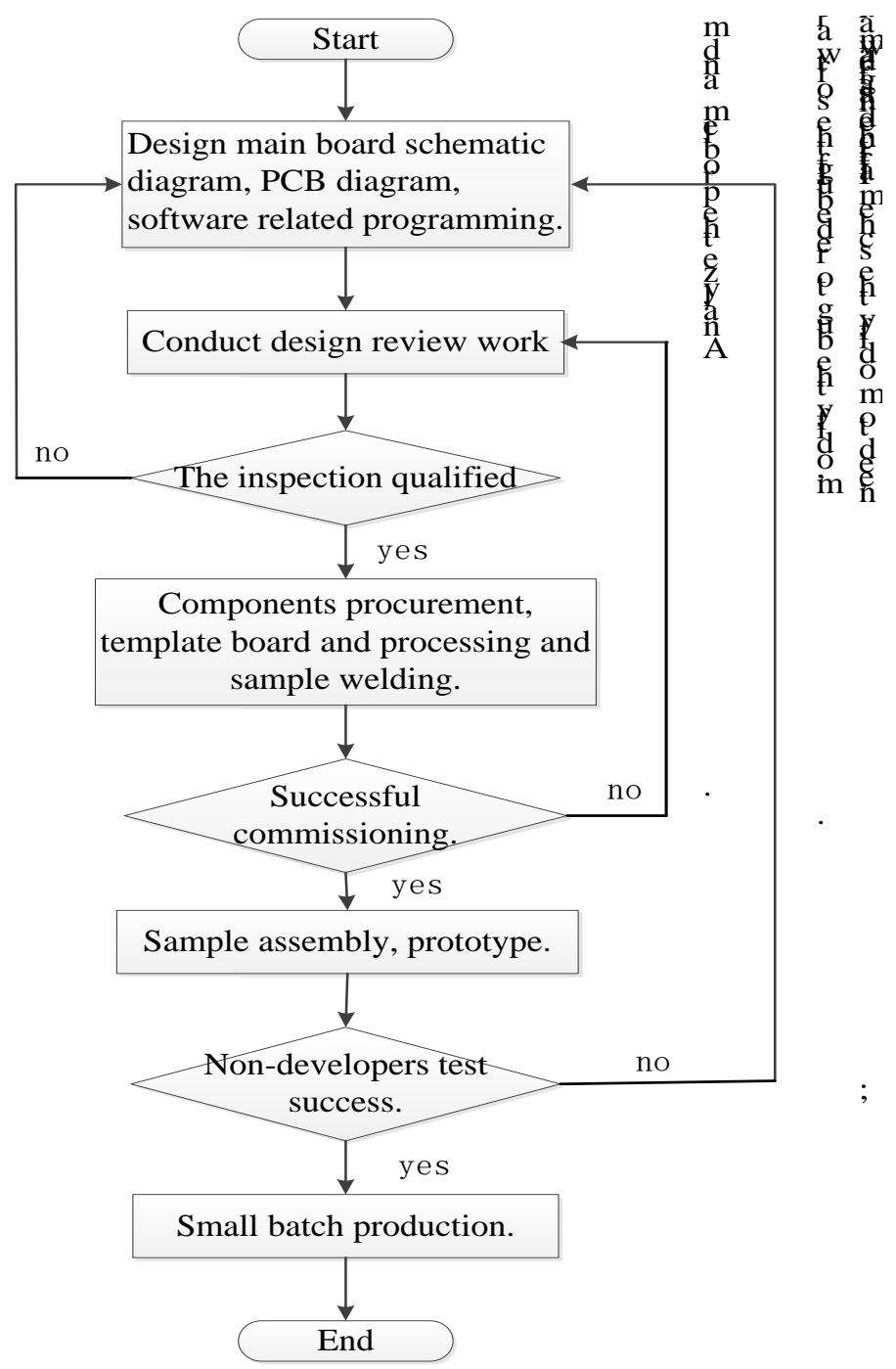

FIG. 1 prototype of intelligent helmet prototype. 


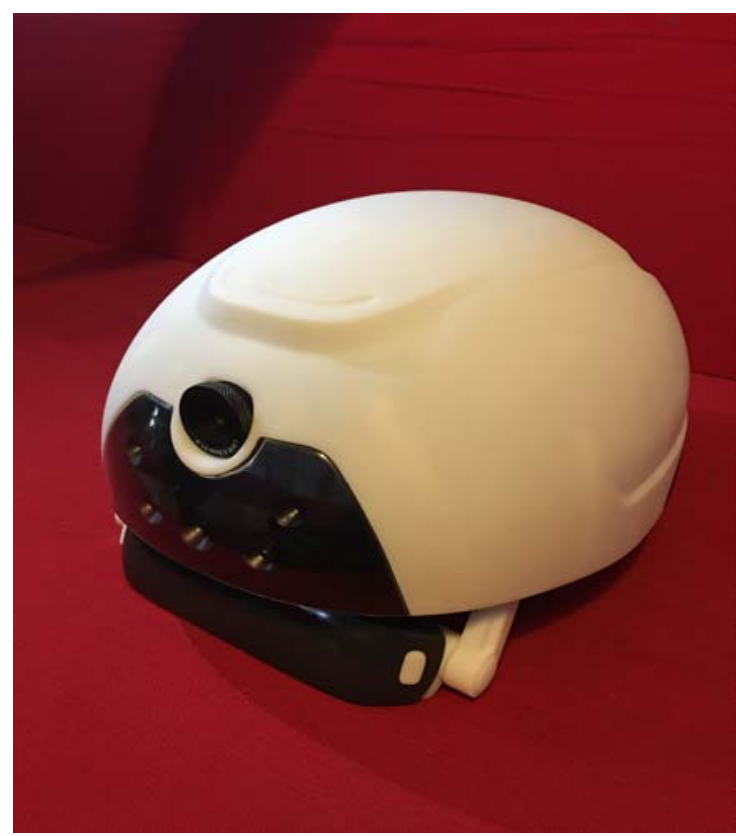

FIG. 2 display of smart helmet prototype.

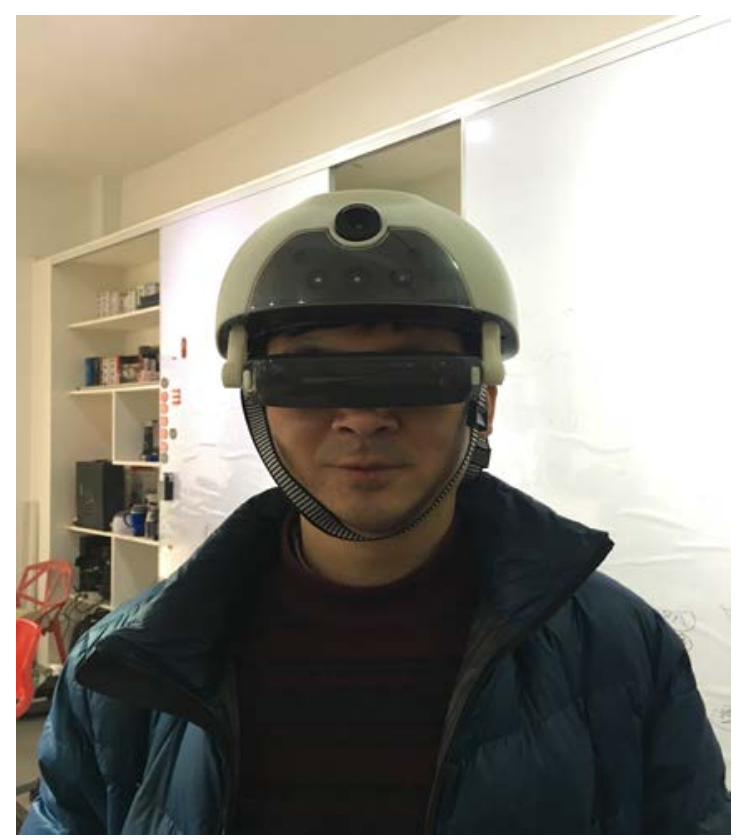

FIG. 3 smart helmet wearing renderings.

\section{Conclusion}

Intelligent wearable device is an important development direction of mobile computing in the future. Smart helmet is one of the important equipment forms of smart wearable devices. It is a comprehensive embodiment of the frontier technology of multi-disciplines.Through the development of the power grid operation intelligent helmet equipment independent intellectual property rights, set up in the field of intelligent wearable hardware professional r\&d team, expand business applications for intelligent wearable technology foundation, leading and promoting the development of relevant industries and technology.

\section{Acknowledgments}

The project was carried out under the strong support of the global energy Internet research institute, a limited company project, with the development of intelligent helmet equipment for independent intellectual property rights. The design process of the smart helmet is mainly described and the finished product of the smart helmet is presented.

\section{References}

[1] Geng li Ming, yan congcong. Design and simulation of PADS 9.5 circuit from introduction to mastery [M]. People's post and telecommunications press, 2015.

[2] Ruan baoxiang, shao xianghua. Industrial design man-machine engineering [M]. Mechanical industry press, 2010.

[3] Zhou meiyu. Application of human engineering in industrial design [M]. China light industry press, 2001. 